\title{
Life cycle analysis of perfluorooctanoic acid (PFOA) and its salts in China
}

\author{
Jing Meng ${ }^{1,2}$, Yonglong Lu ${ }^{1 *}$, Tieyu Wang ${ }^{1}$, Pei Wang ${ }^{1}$, John Giesy ${ }^{3}$, \\ Andrew Sweetman ${ }^{4,5}$ and Qifeng Li \\ 1 \\ State Key Laboratory of Urban and Regional Ecology, Research Center for Eco-Environmental \\ Sciences, Chinese Academy of Sciences, Beijing 100085, China \\ ${ }^{2}$ University of Chinese Academy of Sciences, Beijing 100049, China \\ ${ }^{3}$ Department of Veterinary Biomedical Sciences and Toxicology Centre, University of \\ Saskatchewan, Saskatoon, Saskatchewan, Canada \\ ${ }^{4}$ Centre for Ecology \& Hydrology, Maclean Building, Crowmarsh Gifford \\ Wallingford, Oxon, $O X 108 B B, U K$ \\ ${ }^{5}$ Lancaster Environment Centre, Lancaster University, Lancaster LA1 4YQ, UK \\ *corresponding author: yllu@rcees.ac.cn
}

\begin{abstract}
China has been the largest producer and emitter of perfluorooctnoic acid and its salts (PFOA/PFO). However, the flows of PFOA/PFO from manufacture and application to environment was indistinct. Here a life cycle analysis of PFOA/PFO is presented, in which all major flows of FOA/PFO have been characterized for 2012. Processes related to uses and possible releases of PFOA/PFO include: manufacture and use, waste management, and environmental storage. During manufacture and use,
\end{abstract}


emission from application was the most important $(33.3 \mathrm{t})$, regardless of whether it flowed first to waste treatment facilities or was directly released to environment, followed by manufacture of PFOA/PFO (3.9 t), while flows from service life and end-of-life of consumer products were the lowest (1.5 t). Among five waste treatment routes, flows through wastewater treatment plants (WWTPs) were the highest (28.2 t), followed by releases to environment at $32.4 \mathrm{t}$. Masses of PFOA/PFO emitted were estimated to be $33.5 \mathrm{t}$ to the hydrosphere, $7.2 \mathrm{t}$ to the atmosphere and $1.1 \mathrm{t}$ to soils. Therefore, control measures for reduction of PFOA/PFO should focus on application of PFOA/PFO using reliable alternatives and emission reduction from WWTPs using effective treatment techniques.

\section{Introduction}

Perfluoroalkyl substances (PFASs) are a group of chemicals that have been widely used in various industrial and consumer products, such as carpet, fiber, leather, packaging, paper and textile for more than 60 years (OECD 2013). PFASs have been widely detected in environmental matrices, wildlife and humans, especially perfluorooctane sulfonate (PFOS) and perfluorooctnoic acid (PFOA) (Giesy and Kannan 2001, Giesy and Kannan 2002). Due to their persistence in the environment, hazardous effects and potential accumulation in food webs, PFOS and related compounds have been listed in the Stockholm Convention on Persistent Organic Pollutants (POPS) in 2009 (Wang et al. 2009). Also, to reduce industrial PFOA emission and residual PFOA content in commercial products, the U.S. Environmental 
Protection Agency (USEPA) and the eight major fluoropolymer (FP) and fluorotelomer (FT) manufacturers initiated the voluntary 2010/2015 PFOA Stewardship Program in 2006 (USEPA 2006). In European Union (EU), PFOA and its ammonium salt (APFO) have been identified as persistent, bioaccumulative and toxic (PBT) substances (Vierke et al. 2012, ECHA 2013). For this reason, in 2015, the EU council decided that it should submit a proposal to the Secretariat of the Stockholm Convention suggesting that PFOA and its compounds should be added to Annex A of the Convention.

An inventory of sources and emissions of PFOA/PFO to the environment have been estimated for the globe and China (Wang et al. 2014, Li et al. 2015). Since 2002, there has been a geographical shift of industrial sources of PFOA from primarily North America, Europe and Japan to emerging Asian economies. China has become the largest emitter of PFOA/PFO (REF). To estimate emissions of PFOA/PFO, most assessments have focused on production and industrial applications, while little attention has been paid to treatment of wastes. However, during production and application by industries, most PFOA/PFO is released into waste treatment facilities, which do not directly enter the ambient environment (REF). Therefore, it is essential to develop a substance flow analysis for PFOA/PFO from a life cycle perspective.

Beginning in the 1970s, life cycle analysis (LCA) was developed from analyzing the use of energy to a more comprehensive assessment of movements of masses of materials (Guinee et al. 2011). LCAs for metals, such as anthropogenic nickel, iron, aluminum and chromium have been conducted widely (Johnson et al. 2006, Wang et 
al. 2007, Reck et al. 2008, Chen et al. 2010). However, LCAs for persistent organic pollutants (POPs) are relatively scarce, with a few being conducted for polycyclic aromatic hydrocarbons (PAHs) and polybrominated diphenyl ethers (PBDEs) (Lee et al. 2015, Ventura et al. 2015). For PFOA and PFOS, LCAs have been conducted for Switzerland (FOEN 2009). Since there was no production of PFOA and related compounds in Switzerland, PFOA was mainly released during manufacturing of carpets, aqueous firefighting foams (AFFFs) and textiles. Wastewater was the dominant pathway for emission of PFOA to the environment.

The LCA for PFOA/PFO included three stages: production and use, waste management and storage in the environment. The LCA for PFOA/PFO offers new perspectives on reduction, alternative processes, manufacturing of products, treatment of wastes, and general management of the environment. This assessment has estimated emissions of PFOA/PFO at various stages of the whole life cycle, results of which identify current and future flows of PFOA/PFO in China. Such estimates could further help governmental departments make more detailed assessments of risks and implement realistic policies for controlling releases of PFOA/PFO at each stage of the life cycle. Furthermore, as a signatory to the Stockholm convention, the results of this analysis will help the central government of China meet its obligations under the treaty for any future changes in proposed use and management of PFOA/PFO. Finally, the assessment can serve as a model for other developing countries to apply LCA promulgating policies for their own specific situations. 


\section{Materials and methods}

Identification of sources of PFOA/PFO. Based on estimates of emissions for the globe and China, sources of PFOA/PFO were identified, mainly including synthesis of PFOA/PFO, productions of fluoropolymers (FP), application of aqueous fluoropolymer dispersions (AFDs), industrial processes using perfluorooctane sulfonyl fluoride (POSF)- and fluorotelomer (FT)-based substances and domestic emission during use and disposal of FP-, POSF- and FT-based substances (Prevedouros et al. 2006, Wang et al. 2014, Li et al. 2015). PFOA/PFO as ingredients are released from production of PFOA/PFO and FP, and applications of AFDs, directly as impurities or transformation products from precursors during application of POSF- and FT-based substances. During production and application of relevant substances, some PFOA/PFO can directly enter the environment while some enter via waste treatment facilities. Therefore, waste treatment facilities, such as industrial and domestic wastewater treatment plants (WWTPs) and landfill sites are potential sources of PFOA/PFO to the wider environment (Kim et al. 2012, Taylor et al. 2014, Clarke et al. 2015).

Framework of LCA. The LCA was based on relevant data for PFOA/PFO during 2012. System boundaries ranged from production to environmental storage of PFOA/PFO and included three stages: production and use, waste management and storage in environmental compartments (Fig.1). Waste management included sewage treatment systems, WWTPs, landfill, incineration and stacking. Environmental storage was estimated by information on releases from production, processing and use 
of relevant substances, and waste treatment.

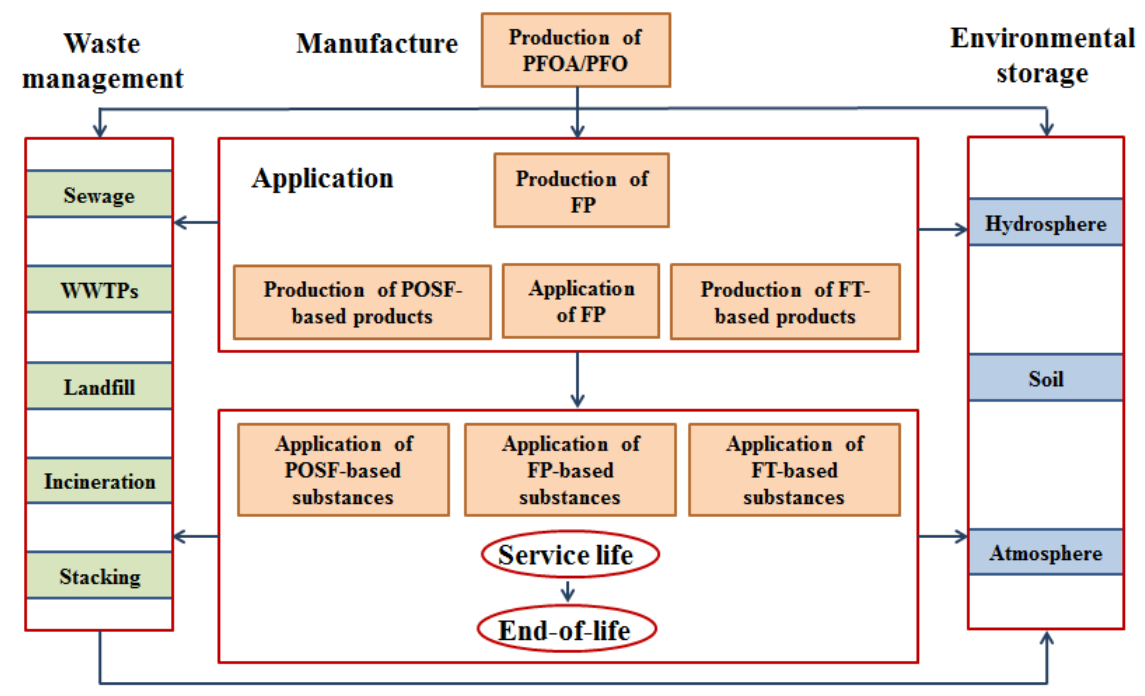

Fig. 1. Sources of PFOA/PFO during the life cycle.

Acquisition of data on production and use. According to the Chinese National $12^{\text {th }}$ Five-year Plan for Fluorine Chemical Industry, in 2010, total production of perfluoroalkyl carboxylic acids (PFCAs) was $80 \mathrm{t}$, most of which was PFOA (CAFSI 2011). In 2012, approximately $90 \mathrm{t}$ of PFOA was produced (Li et al. 2015). In industrial applications, PFOA/PFO and formulated products were used mainly as emulsifiers in suspension and emulsion polymerizations to produce FP. It was reported that $640 \mathrm{~g}$ PFOA/PFO were used to produce $1 \mathrm{t}$ of PTFE in China (Zhou 2002). According to the capacity for production of PTFE in 2012 and forecast for production of FP in 2015, it was estimated that the volume of FP produced in 2012 would be 79,000 t (Table S1). There were three primary types of FP products, including granular (72.8\%), fine powder (19.7\%) and aqueous dispersion $(7.5 \%)$ in China (Wang 2006). Due to high-temperature melting or sintering treatment (usually $>350{ }^{\circ} \mathrm{C}$ ) during application of granular and fine powders, most PFOA/PFO are decomposed and only trace amounts (1-10 ppm) remain in plastics (Cope 2005). 
Therefore, releases via this process to the environment are likely to be negligible. While aqueous fluoropolymer dispersions (AFDs) are almost entirely applied to formulate coating products, during which $26 \%$ of PFOA/PFO residuals are released (FMG 2003).

On average, approximately $150 \mathrm{t}$ of POSF-based substances are produced annually in China, from which approximately 50\% were exported (CAFSI 2013). In addition, $50 \%$ domestic consumption of POSF-based substances were mainly applied in three industrial sectors: metal plating as mist suppressants (20\%), AFFFs as antifoaming surfactants (20\%), and sulfluramid as a raw material (5\%) (Zhang et al. 2012). Application in other industries, such as oil exploitation and semiconductor manufacture, was negligible.

In China, FT-based substances are produced during two processes including: completely domestic production and secondary formulation using imported telomere A. In 2012, domestic manufacturers began to produce FT-based substances, with a total output of 1,500 $\mathrm{t}$ ( $\mathrm{Li}$ et al. 2015). In addition, approximately $600 \mathrm{t}$ of telomer $\mathrm{A}$ were imported annually to process FT-based substances (Chen 2010), with production volume of 3,000 $\mathrm{t}$ (Prevedouros et al. 2006). Almost all of this production was applied as finishing agents in processing of textiles and leather.

Identification of transfer coefficients (TC) and emission factors (EF). In this LCA, TCs and EFs of PFOA/PFO were determined based on information in the literature, from controlled laboratory experiments, reports from the government or organizations and statistical data. Details of TCs and EFs are available in Supporting 
Materials. The transport volume to next stage or environmental release of PFOA/PFO was calculated as follows (Equation 1).

Transport volume (environmental release) of $\mathrm{PFOA} / \mathrm{PFO}=\mathrm{TC}(\mathrm{EF}) \times$ PFOA/PFO input

\section{Results and discussion}

Flows in production and use. Releases of PFOA/PFO during application, which involved production of FP, processing of FP-, POSF- and FT-based substances, were dominant (Fig. 2). Emission during production of PFOA/PFO was relatively higher. Therefore, emissions of PFOA/PFO from production and application by industries was greater than those from domestic sources, including emissions during its service life and end-of-life. Estimates of industrial sources (Fig. S1), included during production of PFOA/PFO, production and processing of FP, use of AFDs and uses of POSF/FT-based substances during industrial processes, were 3.9, 32.9, 0.3 and $0.2 \mathrm{t}$, respectively. These estimates of emissions for China are similar to those reported elsewhere for 2004 to 2012 (Li et al. 2015). PFOA/PFO from industrial sources were released with waste streams, namely exhaust gases, wastewaters and solid wastes. PFOA/PFO released from industrial sources to WWTPs, solid waste and atmosphere were calculated to be $26.8,3.4$ and 7.0 t, respectively (Fig. S1). During production of PFOA/PFO, residues generally entered into WWTPs (3.7 t), accounting for $94.4 \%$ of total wastes. Most PFOA/PFO was used in production of FP with approximately $65.4 \%$ of annual usage. China was the largest producer of FP with 3.7\% net export (GACC 
2013). During production of FP, 55.6\% PFOA/PFO was discharged with wastes, especially wastewater (23.0 t), followed by waste gases $(6.6 \mathrm{t})$. When FP were used in commercial products, almost all PFOA/PFO were decomposed in FP powder, with approximately 26\% PFOA/PFO emitted in AFDs. As a result, $0.31 \mathrm{t} \mathrm{PFOA} / \mathrm{PFO}$ were emitted during use of FP. During production and application of POSF-based substances, $0.016 \mathrm{t}$ of PFOA/PFO were discharged with wastes. During production and application of FT-based substances, mainly textiles and leather, PFOA/PFO with a volume of $0.15 \mathrm{t}$ was discharged to the atmosphere.

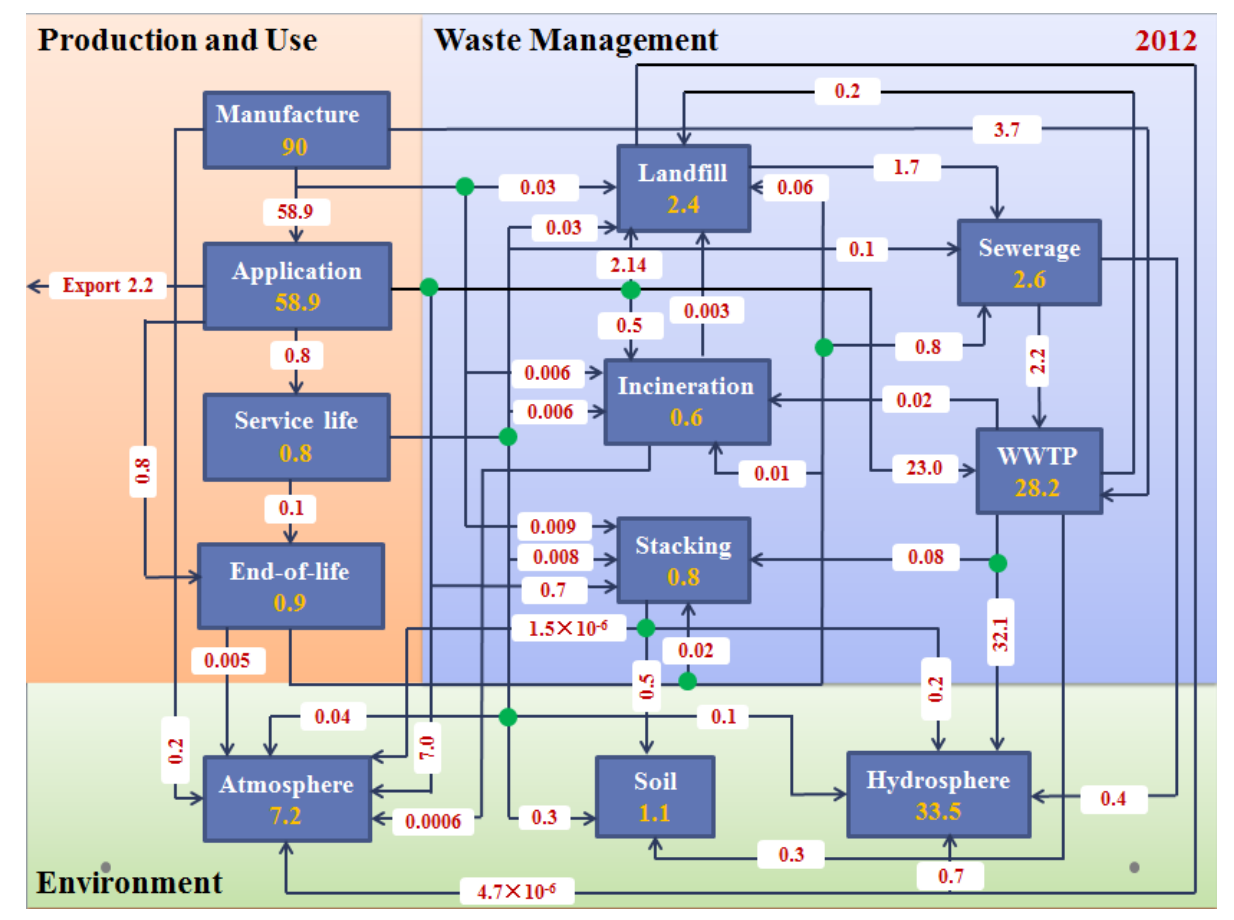

Fig.2. Life cycle analysis of PFOA/PFO in 2012.

Note: flows between processes are reported in ton.

After PFOA/PFO related compounds were used to manufacture various consumer products, residual PFOA/PFO remained in the products. During their use and disposal, PFOA/PFO were released, which was defined as domestic sources. PFOA/PFO residues were estimated using a bottom-up method (Li et al. 2015). 
Among applications of these three kinds of PFOA/PFO related substances, residues of PFOA/PFO in POSF-based substances were greatest, including those in AFFF and sulfluramid, followed by those in textiles and leather, while residues of PFOA/PFO in coatings were the lowest (Fig. S2). AFFF produced in China were consumed domestically (Yv 2010), so it was assumed that no AFFF were exported and also that no AFFF was imported. China is a major exporter of textiles, with approximately $30 \%$ of the total production of textiles being exported (Chen 2014). Due to unavailable data on exports and imports of other products, it was assumed that coating products, sulfluramid and leather were all used in the domestic market. It was assumed that during service life, PFOA/PFO in various products entered the environment. Due to their use in high-temperature of coating products, most $\mathrm{PFOA} / \mathrm{PFO}$ are released to the atmosphere, while PFOA/PFO in textiles and leather mainly entered sewage treatment facilities. After use of AFFF, PFOA/PFO directly entered into hydrosphere and soil. It was assumed that PFOA/PFO along with use of sulfluramid entered into soil. During the service lives of these products (Fig. S2), $0.67 \mathrm{t}$ of PFOA/PFO were discharged into waste treatment facilities and the environment. PFOA/PFO from coatings, AFFF and sulfluramid $(0.47 \mathrm{t})$ were directly emitted into the environment. Most PFOA/PFO $(0.13 \mathrm{t})$ from textiles and leather were emitted in sewage, followed by those emitted with solid waste $(0.04 \mathrm{t})$. Treatment of waste or expired products resulted in $0.88 \mathrm{t}$ of PFOA/PFO being emitted with waste streams. PFOA/PFO from expired AFFF $(0.78 \mathrm{t})$ was the greatest, with mass of PFOA/PFO entering sewage directly. For other products, including used coating products, textiles and leather, all PFOA/PFO (0.1 t $)$ 
was released with solid waste. Most PFOA/PFO in commercial products entered the environment associated with wastes. Therefore, management and treatment of used or expired products was a significant portion of the mass of PFOA/PFO entering the environment and a prime target for reduction of overall loadings to the environment.

Flows in waste management. Among the five routes for treatment of wastes containing PFOA/PFO, most of the waste material was generated during manufacture and application of PFOA/PFO and wastes generated during the service life and end-of-life of consumer products (Fig. 2). Waste management was primarily via solid waste and wastewater, and thus most PFOA/PFO were stored at WWTPs and landfill sites. Wastewater from industries was released to WWTPs, while that from domestic applications entered the sewage system and then passed through WWTPs. As a result, most PFOA/PFO (1.4 t $)$ in sewage and $26.7 \mathrm{t}$ of PFOA/PFO from industrial processes did not enter the environment directly, but rather entered WWTPs, where it received some form of treatment before entering the ambient environment. In WWTP, pollutants can be released to the environment via treated effluent or sludge. Results of previous studies have found that conventional treatment was not effective at removing PFOA, and that concentrations of PFOA even increased due to biodegradation of precursors (Guo et al. 2010), which resulted in greater emissions to the hydrosphere (32.1 t), while PFOA/PFO in sewage sludge was mainly transferred to landfills $(0.2 \mathrm{t})$ or applied to agricultural soils $(0.3 \mathrm{t})$.

Since there are no data concerning the disposal of solid wastes, estimation of this portion of the flow was based on treatment of domestic solid waste. For treatment of 
solid waste and sewage sludge, incineration was found to be the primary disposal route in other countries. Based on that fact that most PFOA/PFO would likely be degraded, which was different from the situation in China. In Europe, the majority of sludge was disposed of to landfill $(\sim 57 \%)$, followed by application as a fertilizer to land. Application of sludge in agriculture varied greatly among countries. For example, in Spain $65 \%$ was disposed of in that manner, while in Germany it was $30 \%$ (Gomez-Canela et al. 2012). Application of sewage sludge to land poses risks to soils. Results of a previous study showed that concentrations of all PFASs in soil increased linearly as a function of rates of loading of sludge (Sepulvado et al. 2011).

Results of more recent studies have demonstrated that landfills were sources of semivolatile PFASs to surrounding air and nonvolatile PFASs to landfill leachates (Ahrens et al. 2011, Huset et al. 2011, Benskin et al. 2012). Concentrations of PFOA in municipal landfill leachates in Germany ranged from $<0.40$ to $926 \mathrm{ng} / \mathrm{L}$ (Busch et al. 2010). Concentrations of PFOA in landfill leachate in the USA showed a mean concentration of $820 \mathrm{ng} / \mathrm{L}(177 \sim 2500 \mathrm{ng} / \mathrm{L})$ (Clarke et al. 2015). In China, landfill is the main method the for treatment of solid wastes, with agricultural application being common for sewage sludge, which resulted in greater releases to soil. When concentrations of PFASs were measured at landfill sites in five relatively developed cities in China, concentrations in leachate ranged from 7,280 to $292,000 \mathrm{ng} / \mathrm{L}$. National leakage of PFASs to groundwater from landfill leachate was estimated to be $3,110 \mathrm{~kg} / \mathrm{y}$, which indicated risks to groundwater (Yan et al. 2015). When wastes containing PFOA/PFO were placed into landfills, most of the PFOA/PFO entered into 
leachate, which resulted in $1.7 \mathrm{t}$ of PFOA/PFO going into sewage (von Arx 2006). At stacking sites, it was assumed that leachate would be generated in quanitities similar to landfill sites, even though the fate of the leachates would have been different. Leachate from wastes at stacking sites would directly flow into soils $(0.5 \mathrm{t})$ and be released to the hydrosphere $(0.2 \mathrm{t})$, so stacking sites were considered to be important sources of emissions. At incineration sites PFOA/PFO should mostly degrade if the temperatures were sufficiently high. It was estimated that a relatively small amount (0.003 t) would be released and then enter into other pathways. Therefore, besides storage in WWTPs, landfills and stacking sites are also significant sources of PFOA/PFO. This might be specific to China and different from other countries.

Storage in the environment. In China, storage of PFOA/PFO in soils, the hydrosphere and atmosphere were estimated to be $1.1,33.5$ and $7.0 \mathrm{t}$, respectively (Fig. 2). PFOA/PFO existed predominantly in the hydrosphere and atmosphere, while little was stored in soils. For PFOA in China, the hydrosphere was also the largest sink. One of the only other assessments of flows of PFOA/PFO in the environment, conducted in Switzerland, suggested that the greatest proportion of PFOA/PFO (33 kg) was in the hydrosphere, with $0.2 \mathrm{~kg}$ in the atmosphere and $2 \mathrm{~kg}$ in soil (FOEN 2009). One significant difference between Switzerland and China is that there was no manufacture of PFOA/PFO and few applications of related products in Switzerland. Most PFOA/PFO in the atmosphere $(7.0$ t) was released from applications, contributing 97.2\%. Most of the PFOA/PFO in the hydrosphere $(32.1 \mathrm{t})$ originated from WWTPs, which was estimated to contribute $95.8 \%$ of the PFOA/PFO 
released to the hydrosphere. Contributions from other processes or sites to soil were relatively similar, with $0.5 \mathrm{t}$ from stacking sites, $0.3 \mathrm{t}$ during service life of products and $0.3 \mathrm{t}$ from treatment of sewage sludge. Contributions from industrial and domestic applications differed greatly. Most PFOA/PFO was released in one way or another from industrial sources, which accounted for $98.6 \%$ of the total, most of which was released during production of FP. PFOA/PFO from solid waste at stacking sites and application of sewage sludge in agriculture resulted in more PFOA/PFO being released to soil in China compared to that in other countries.

Inventories can be based on absolute masses, expressed as concentrations in each compartment or in relative terms based on proportions flowing along each pathway. Although emissions to environmental media were estimated, due to transport and transformation in these three media, relative concentrations of PFOA in the environment may be different. For example, PFAAs in air might 'flow' into soil and water via wet and dry deposition. Concentrations of PFAAs have been measured for the environment in China, especially PFOA and PFOS (Wang et al. 2015). Compared with PFOS, which is already listed in Appendix A of the Stockholm Convention, current concentrations of PFOA were even higher in fresh water, especially in severely contaminated rivers, such as Daling River, Liaohe River, Hanjiang River, Yangtze River and Huaihe River. Concentrations of PFOA in lakes of China, such as Taihu Lake, Tangxun Lake and East Lake, were similar to those reported for PFOS in the same lakes. PFAAs, especially PFOA and PFOS with concentrations ranging from 
tens of picograms to thousands of nanograms per liter of water, were widespread in the hydrosphere. Concentrations of PFOA in soil have been measured primarily in coastal areas of the Bohai Sea (Pan et al. 2011, Wang et al. 2012). Concentrations of PFOA and PFOS were the predominant PFAAs in soils along the Bohai Sea, with concentrations of PFOA ranging from n.d. to $13 \mathrm{ng} / \mathrm{g}$ (Meng et al. 2015). Concentrations of PFOA in soils in the vicinity of Shanghai were relatively high, ranging from 3.3 to $48 \mathrm{ng} / \mathrm{g}$ ( $\mathrm{Li}$ et al. 2010). While measurements of PFOA in air were few, one campaign conducted in Shenzhen detected PFOA in all samples, with concentrations ranging from 1.5 to $15 \mathrm{pg} / \mathrm{m}^{3}$ (Liu et al. 2015).

Alternatives or mitigation technologies for PFOA/PFO. Through the LCA for production and use of PFOA/PFO and ultimately dispersion in the environment, it is possible to design more effective programs of monitoring and exposure assessment to determine which receptors would be most at risk (Fig. 3). This information can also be used to mitigate risks by minimizing releases, including which applications of PFOA/PFO in various industries and which treatment of wastewater and solid waste result in the highest exposures. Finally, the absolute and relative masses predicted to move along specific pathways allows for more effective mitigation of releases.

During production of PFOA/PFO, the most effective currently available technology to reduce releases is vacuum distillation. While this would reduce releases, the PFOA reclaimed could be reused for production of organic fluoride, such as PTFE, which is a improved method compared to primary production using ECF (Tang 2009). There are several alternative ways to minimize releases of PFOA during the 
application of PFOA/PFO, and some technologies are more effective than others (REF). Owing to the presence of biological residues and slow degradation of C8 products, development of alternatives has been focused on two technologies: use of low-carbon fluoride products and alternatives with heteroatoms, such as oxygen or chlorine (Lu 2011). Currently 3M company has implemented use of perfluorobutane sulfonate (PFBS), which is a shorter-chain replacement for PFOS (3M 2007). Dupont uses telomerization to produce perfluorinated alkyl monomers, mainly C6-based products to replace C8 products (Gu 2009). Perfluoropolyethers (PFPEs) have also been considered to be effective alternatives. For example, Dupont uses PFPEs or their salts as emulsifiers during production of PTFE and Solvay Solexis uses PFPEs in food packaging materials. PFPEs are absorbed less efficiently and have a shorter half-life and are not degraded into PFOA/PFOS (REF) and have therefore been used in commercial applications. However, some reports have indicated global warming potential of PFPEs due to the presence of C-O and C-F bonds (Young et al. 2006). With increased production and application of PFPEs they may present a risk to global warming. In the firefighting industry, fluorinated surfactants produced using the telomerization process were demonstrated to be effective alternatives to PFOS and PFOA. However, their risks to the environment due to widespread use in AFFF have not yet been assessed. Firefighting technologies using compressed air/foam have also been considered feasible (Tian 2009). For metal plating polyfluorinated ether sulfonates (PFAESs) have been used as alternatives for PFOS. A 6:2 chlorinated ether sulfonate (6:2 Cl-PFAES) with the commercial name F-53B has been used in China 
since the 1970s (Ruan et al. 2015). F-53B has moderate toxicity and is as persistent as PFOS. Concentrations of F-53B were similar to those of PFOS in the ambient environment around metal plating factories (Wang et al. 2013). Although F-53B have been used for many years, the impact has yet to be fully assessed so increasing their application in replacement of PFOS should be only advocated with caution.

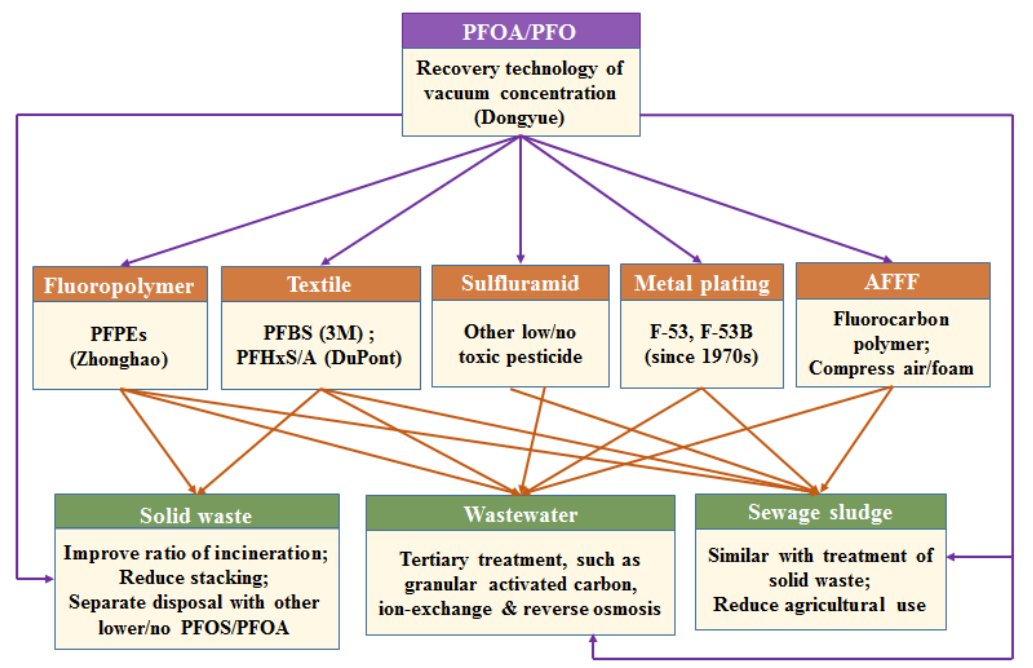

Fig.3. Alternatives or mitigation techniques for PFOA/PFO.

Note: "purple box" represents techniques used during manufacture of PFOA/PFO; "orange box" represents alternatives or mitigation techniques during application of PFOA/PFO; "green box" represents mitigation techniques during waste treatment; "bracket" is representative enterprise.

Apart from technologies for reducing emissions or developing alternatives for use in applications, some mitigating measures can also be implemented during waste treatment. WWTPs were the most important receiving and discharging sites for PFOA/PFO due to poor efficiencies of removal by conventional wastewater treatment techniques and the water solubility of PFOS. Due to the relatively polar structure and thus greater water solubility of PFOS, tertiary treatment techniques, such as granular activated carbon, ion-exchange and reverse osmosis have limited effectiveness for its 
removal (Vecitis et al. 2009). Incineration is the most effective treatment to remove PFOS in solid waste or sewage sludge (REF). As long as the minimum temperature is maintained, this method is effective and results in complete mineralization of PFOS. For China, reduction in use of wastewater for irrigation and sludge for agricultural use were the most cost-effective measures that could be taken to reduce accumulation of PFOA in soils.

Uncertainty analysis. Uncertainty in the LCA for PFOA originates primarily from the limited data available for some of the required parameters such as production and consumption, as well as determination of transfer coefficients and emission factors. Transfer coefficients and emission factors were mainly determined through experimental data, with consideration of the practical situation in China. This, of course, made the assessment specific to processes and storage in environmental media for China. Since there were no related statistical or empirical experimental data on treatment of industrial solid waste, it was assumed that industrial solid waste was treated the same as domestic solid waste. Since there was no information on total amounts of PFOA or precursors from different products, a bottom-up method was applied for estimation of emissions of PFOA from domestic sources in China.

\section{Acknowledgement:}

This study was supported by the National Natural Science Foundation of China under Grant No. 414201040045 and No. 41371488, the International Scientific Cooperation Program with Grant No. 2012DFA91150, and the Key Project of the 
Chinese Academy of Sciences under Grant No.KZZD-EW-TZ-12. Prof. Giesy was supported by the Canada Research Chair program, the Einstein Professorship Program of the Chinese Academy of Sciences and a Visiting Distinguished Professorship in the School of Biological Sciences of the University of Hong Kong. 


\section{References}

3M, C. (2007). Technical data bulletin, enviromental, health, safety, and regulatory (EHSR) profile of per -fluoro -butane sulfonate (PFBS).

Ahrens, L., M. Shoeib, T. Harner, S. C. Lee, R. Guo and E. J. Reiner (2011). "Wastewater Treatment Plant and Landfills as Sources of Polyfluoroalkyl Compounds to the Atmosphere." Environmental Science \& Technology 45(19): 8098-8105.

Benskin, J. P., B. Li, M. G. Ikonomou, J. R. Grace and L. Y. Li (2012). "Per- and Polyfluoroalkyl Substances in Landfill Leachate: Patterns, Time Trends, and Sources." Environmental Science \& Technology 46(21): 11532-11540.

Busch, J., L. Ahrens, R. Sturm and R. Ebinghaus (2010). "Polyfluoroalkyl compounds in landfill leachates." Environmental Pollution 158(5): 1467-1471.

CAFSI (2011). National 12th Five-year Plan for Fluorine Chemical Industry. C. A. o. F. a. S. Industry, China Association of Fluorine and Silicone Industry.

CAFSI (2013). Inventory of perfluorooctane sulfonic acid (PFOS) and related chemicals (in Chinese). Beijing China Assocition of Fluorine and Silicone Industry (CAFSI).

Chen, H. (2010). "Competitive strength and market analysis of electronic chemicals and special gas containing fluorine (in Chinese) " Chemical production and technology 17(6): 1-8.

Chen, W., L. Shi and Y. Qian (2010). "Substance flow analysis of aluminium in mainland China for 2001, 2004 and 2007: Exploring its initial sources, eventual sinks and the pathways linking them." Resources Conservation and Recycling 54(9): 557-570.

Chen, Y. (2014). "Current status of textile trade in China." Modern Business(29).

Clarke, B. O., T. Anumol, M. Barlaz and S. A. Snyder (2015). "Investigating landfill leachate as a source of trace organic pollutants." Chemosphere 127: 269-275.

Cope, C. S. (2005). Measurement of residual C-8 fluorosurfactant levels on various products (226-2533). US EPA Administration Record 226: 1-3.

ECHA. (2013). "Candidate List of Substances of Very High Concern for Authorisation."

FMG (2003). Final Report of Dispersion Processor Material Balance Project (US EPA OPPT 2003-0012-0900, 0901, 0902, 0903, 0904). Washington, DC, Fluoropolymer Manufactures Group (FMG).

FOEN (2009). Substance flow analysis of PFOS and PFOA for Switzerland. Bern, Federal Office for the Environment, the Federal Department of Environment, Transport, Energy and Communications.

GACC (2013). China customs statistical yearbook, General Administration of Customs of China.

Giesy, J. P. and K. Kannan (2001). "Global distribution of perfluorooctane sulfonate in wildlife." Environmental Science \& Technology 35(7): 1339-1342.

Giesy, J. P. and K. Kannan (2002). "Perfluorochemical surfactants in the environment." Environmental Science \& Technology 36(7): 146A-152A.

Gomez-Canela, C., J. A. C. Barth and S. Lacorte (2012). "Occurrence and fate of perfluorinated compounds in sewage sludge from Spain and Germany." Environmental Science and Pollution Research 19(9): 4109-4119.

Gu, W. F., DD; Su, XL (2009). "Reserch progress of PFOA substitutes." Organo-Fluorine Industry(3): 28-30.

Guinee, J. B., R. Heijungs, G. Huppes, A. Zamagni, P. Masoni, R. Buonamici, T. Ekvall and T. Rydberg (2011). "Life Cycle Assessment: Past, Present, and Futures." Environmental Science \& Technology 
45(1): 90-96.

Guo, R., W.-J. Sim, E.-S. Lee, J.-H. Lee and J.-E. Oh (2010). "Evaluation of the fate of perfluoroalkyl compounds in wastewater treatment plants." Water Research 44(11): 3476-3486.

Huset, C. A., M. A. Barlaz, D. F. Barofsky and J. A. Field (2011). "Quantitative determination of fluorochemicals in municipal landfill leachates." Chemosphere 82(10): 1380-1386.

Johnson, J., L. Schewel and T. E. Graedel (2006). "The contemporary anthropogenic chromium cycle." Environmental Science \& Technology 40(22): 7060-7069.

Kim, S.-K., J.-K. Im, Y.-M. Kang, S.-Y. Jung, Y. L. Kho and K.-D. Zoh (2012). "Wastewater treatment plants (WWTPs)-derived national discharge loads of perfluorinated compounds (PFCs)." Journal of Hazardous Materials 201: 82-91.

Lee, S., Y.-C. Jang, J.-G. Kim, J.-E. Park, Y.-Y. Kang, W.-I. Kim and S.-K. Shin (2015). "Static and dynamic flow analysis of PBDEs in plastics from used arid end-of-life TVs and computer monitors by life cycle in Korea." Science of the Total Environment 506: 76-85.

Li, F., C. Zhang, Y. Qu, J. Chen, L. Chen, Y. Liu and Q. Zhou (2010). "Quantitative characterization of short- and long-chain perfluorinated acids in solid matrices in Shanghai, China." Science of the Total Environment 408(3): 617-623.

Li, L., Z. Zhai, J. Liu and J. Hu (2015). "Estimating industrial and domestic environmental releases of perfluorooctanoic acid and its salts in China from 2004 to 2012." Chemosphere 129: 100-109.

Liu, B., H. Zhang, D. Yao, J. Li, L. Xie, X. Wang, Y. Wang, G. Liu and B. Yang (2015). "Perfluorinated compounds (PFCs) in the atmosphere of Shenzhen, China: Spatial distribution, sources and health risk assessment." Chemosphere 138: 511-518.

Lu, W. Z., BB; Zhu, Y; Gu, WY (2011). "Study on the PFOA Substitute and its Application in the Polymerization of Fluoroelastomer." Organo-Fluorine Industry(2): 20-23.

Meng, J., T. Wang, P. Wang, Y. Zhang, Q. Li, Y. Lu and J. P. Giesy (2015). "Are levels of perfluoroalkyl substances in soil related to urbanization in rapidly developing coastal areas in North China?" Environmental Pollution 199: 102-109.

OECD (2013). PFCs: Outcome of the 2009 survey. Survey on the Production, Use and Release of PFOS, PFAS, PFOA, PFCA, Their Related Substances and Products mixtures Containing These Substances. O. $\mathrm{f}$. E. C.-o. a. Development. Paris.

Pan, Y., Y. Shi, J. Wang, X. Jin and Y. Cai (2011). "Pilot Investigation of Perfluorinated Compounds in River Water, Sediment, Soil and Fish in Tianjin, China." Bulletin of Environmental Contamination and Toxicology 87(2): 152-157.

Prevedouros, K., I. T. Cousins, R. C. Buck and S. H. Korzeniowski (2006). "Sources, fate and transport of perfluorocarboxylates." Environmental Science \& Technology 40(1): 32-44.

Reck, B. K., D. B. Mueller, K. Rostkowski and T. E. Graedel (2008). "Anthropogenic nickel cycle: Insights into use, trade, and recycling." Environmental Science \& Technology 42(9): 3394-3400.

Ruan, T., Y. Lin, W. Thanh, R. Liu and G. Jiang (2015). "Identification of Novel Polyfluorinated Ether Sulfonates as PFOS Alternatives in Municipal Sewage Sludge in China." Environmental Science \& Technology 49(11): 6519-6527.

Sepulvado, J. G., A. C. Blaine, L. S. Hundal and C. P. Higgins (2011). "Occurrence and Fate of Perfluorochemicals in Soil Following the Land Application of Municipal Biosolids." Environmental Science \& Technology 45(19): 8106-8112.

Tang, Y. X., P; Zhou, XD (2009). "Recovery and application of perfluorooctanoic acid (PFOA)." Organo-Fluorine Industry(4): 43-47. 
Taylor, P. H., T. Yamada, R. C. Striebich, J. L. Graham and R. J. Giraud (2014). "Investigation of waste incineration of fluorotelomer-based polymers as a potential source of PFOA in the environment." Chemosphere 110: 17-22.

Tian, L. W. P. F. X. Z., S; Bao, ZM (2009). Basic situation analysis and countermeasure of PFOS in firefighting industry Persistent organic pollutants forum 2009 and the fourth session of the national symposium on persistent organic pollutants. Ningbo, China: 234-235.

USEPA. (2006). "2010/2015 PFOA Stewardship Program."

Vecitis, C. D., H. Park, J. Cheng, B. T. Mader and M. R. Hoffmann (2009). "Treatment technologies for aqueous perfluorooctanesulfonate (PFOS) and perfluorooctanoate (PFOA)." Frontiers of Environmental Science \& Engineering in China 3(2): 129-151.

Ventura, A., T. Lorino and L. Le Guen (2015). "Modeling of Polycyclic Aromatic Hydrocarbons stack emissions from a hot mix asphalt plant for gate-to-gate Life Cycle Inventory." Journal of Cleaner Production 93: 151-158.

Vierke, L., C. Staude, A. Biegel-Engler, W. Drost and C. Schulte (2012). "Perfluorooctanoic acid (PFOA) - main concerns and regulatory developments in Europe from an environmental point of view." Environmental Sciences Europe 24(1): 1-11.

Wang, J. (2006). "Production and import status of PTFE in China (in Chinese)." Organo-Fluorine Industry(1): 19-20.

Wang, S., J. Huang, Y. Yang, Y. Hui, Y. Ge, T. Larssen, G. Yu, S. Deng, B. Wang and C. Harman (2013). "First Report of a Chinese PFOS Alternative Overlooked for 30 Years: Its Toxicity, Persistence, and Presence in the Environment." Environmental Science \& Technology 47(18): 10163-10170.

Wang, T., Y. Lu, C. Chen, J. E. Naile, J. S. Khim and J. P. Giesy (2012). "Perfluorinated compounds in a coastal industrial area of Tianjin, China." Environmental Geochemistry and Health 34(3): 301-311.

Wang, T., D. B. Mueller and T. E. Graedel (2007). "Forging the anthropogenic iron cycle." Environmental Science \& Technology 41(14): 5120-5129.

Wang, T., P. Wang, J. Meng, S. Liu, Y. Lu, J. S. Khim and J. P. Giesy (2015). "A review of sources, multimedia distribution and health risks of perfluoroalkyl acids (PFAAs) in China." Chemosphere 129: 87-99.

Wang, T., Y. Wang, C. Liao, Y. Cai and G. Jiang (2009). "Perspectives on the Inclusion of Perfluorooctane Sulfonate into the Stockholm Convention on Persistent Organic Pollutants." Environmental Science \& Technology 43(14): 5171-5175.

Wang, Z., I. T. Cousins, M. Scheringer, R. C. Buck and K. Hungerbuehler (2014). "Global emission inventories for C-4-C-14 perfluoroalkyl carboxylic acid (PFCA) homologues from 1951 to 2030, Part I: production and emissions from quantifiable sources." Environment International 70: 62-75.

Yan, H., I. T. Cousins, C. Zhang and Q. Zhou (2015). "Perfluoroalkyl acids in municipal landfill leachates from China: Occurrence, fate during leachate treatment and potential impact on groundwater." Science of the Total Environment 524: 23-31.

Young, C. J., M. D. Hurley, T. J. Wallington and S. A. Mabury (2006). "Atmospheric lifetime and global warming potential of a perfluoropolyether." Environmental Science \& Technology 40(7): 2242-2246.

Yv, W. W., PX; Tian, L; Fu, XC; Bao, ZM (2010). "The lastest information of the Stockholm Convention and introduction of the using of PFOS in China fire industry." Fire Science and Technology 29(6): 513-515.

Zhang, L., J. Liu, J. Hu, C. Liu, W. Guo, Q. Wang and H. Wang (2012). "The inventory of sources, environmental releases and risk assessment for perfluorooctane sulfonate in China." Environmental 
Pollution 165: 193-198.

Zhou, Y. H., JingYan; Xue, XueJia (2002). "Preperation and performance measurement of organo-fluorine textile finishing agent (in Chinese)." Fine Chemistry(19): 71-77. 